\title{
Colorectal Cancer Risk Factors: A Study of Knowledge, Attitude and Practice Among Adults in Riyadh, Saudi Arabia
}

\author{
Osama Al Wutayd ${ }^{1}$, Fahad Alamri ${ }^{2, ~ *}$, Arwa Mohammed Ali ${ }^{3,4}$, Kassim Abdelazeem Kassim², \\ Ahmed Khair Ibrahim ${ }^{5}$ \\ ${ }^{1}$ Community Medicine Department, King Saud University, Riyadh, Saudi Arabia \\ ${ }^{2}$ Deputy Ministry for Public Health, Assistant Deputy for Primary Health Care, Ministry of Health, Riyadh, Saudi Arabia \\ ${ }^{3}$ Medical Oncology Department, King Khalid University Hospitals, King Saud University, Riyadh, Saudi Arabia \\ ${ }^{4}$ Medical Oncology Department, South Egypt Cancer Institute, Assiut University, Asyut, Egypt \\ ${ }^{5}$ Public Health \& Community Medicine School, Faculty of Medicine, Assiut University, Asyut, Egypt
}

\section{Email address:}

fahadamri@yahoo.com(F. Alamri)

\section{To cite this article:}

Osama Al-Wutayd, Fahad Alamri, Arwa Mohammed Ali, Kassim Abdelazeem Kassim, Ahmed Khair Ibrahim. Colorectal Cancer Risk Factors: A Study of Knowledge, Attitude, and Practice Among Adults in Riyadh, Saudi Arabia. Cancer Research Journal. Vol. 3, No. 5 ,2015, pp. 94-99. doi: 10.11648/j.crj.20150305.11

\begin{abstract}
Background: Colorectal cancer ranked the second cancers in the Kingdom of Saudi Arabia, accounting for 10.4\% of all newly diagnosed cancers in 2010. There are several risk factors affecting the incidence of colorectal cancer where some factors influence the risk more than others. Colorectal cancer is easily preventable through screening which can detect the disease in its early stages and improve survival rates. Aim: We aimed to determine the level of knowledge, attitude and practice of colorectal cancer and its risk factors among mall adult visitors in Riyadh, Saudi Arabia. Methods: Across-sectional study was conducted in five randomly selected malls (120 from each mall were randomly recruited), using a modified validated questionnaire. Means, standard deviations, frequencies, percentages were calculated for the differences in the level of awareness among the study participants. Results: The response rate was 70\% (55\% were females and $45 \%$ were males). More than one-third of the participants identified cigarette smoking, family history of colorectal cancer and inflammatory bowel disease as risk factors for the disease. In addition, the majority of responses reported that screening tools are useful in colorectal cancer prevention and control. Conclusion: Implementation of awareness programs is needed to establish a strong link between the public, health centers and educational institutions to increase the level of awareness of colorectal cancer.
\end{abstract}

Keywords: Colorectal Cancer, Screening, Awareness, Risk Factors

\section{Introduction}

Colorectal cancer (CRC) was ranked the second most common cause of female cancer and the third most common cancer in males in the year 2012, globally [1]. Moreover, CRC was ranked as the fourth most common leading cause of cancer deaths after lung, stomach, and liver cancer [2]. Higher rates of CRC were reported in developed countries compared with developing ones [3]. CRC incidence rates varied widely over the world [4]. The highest incidence rates were reported in Australia, New Zealand, Europe and the USA while the lowest rates were found in Africa and South-Central Asia [4]. According to cancer statistics, an estimated $6 \%$ of the current global population will develop CRC anytime during their life and half of them will die because of the disease [5]. In the USA, CRC has been identified as the third most common cancer afflicting people [6].

In Asia, a review of literature reported that there was a steady increase in the incidence rates of CRC as well as its mortality in many countries e.g. Singapore, Japan and South Korea [7]. Over the past few decades, there was an estimated 2-4 fold increase in the incidence of the disease [7]. However, it was found that public awareness and heath authority support were inadequate for proper prevention an prompt detection[8].

In Saudi Arabia, the incidence of CRC showed a steady increase in the period between 2001 and 2006, the number of CRC confirmed cases increases, for both genders and in 
different age-groups, from 800 in the year 2001 to 1800 in the year 2006 [9]. Saudi Cancer Registry (SCR) issued its $14^{\text {th }}$ cancer incidence report in the year 2014 whereby the newly diagnosed CRC cases were 1033 representing 10.4\% of all cancers and placed $2^{\text {nd }}$ after breast cancer among Saudi cancer patients. It was ranked the $1^{\text {st }}$ among all male malignancies $(11.8 \%)$ and the $2^{\text {nd }}$ for females $(10.2 \%)$ [10].

Among all cancers, CRC with its rapidly increasing incidence rates, long time between early and advanced stages and long lead-time (diagnosis by screening and confirmatory diagnosis) is considered ideal for screening [11-13]. Various methods of CRC screening were developed for early detection and proper diagnosis, hence early management and better prognosis [14]. These screening tools included; non-invasive methods such as stool DNA and fecal occult blood testing (FOBT) and potentially invasive methods such as colonoscopy $[15,16]$.

CRC risk factors included age of patient; most national guidelines recommended screening for those aged 50 years and older [2]. Other risk factors included family history, obesity and metabolic syndrome. Moreover, first-degree relatives of CRC patient have a 2-fold increased risk and siblings of subjects with advanced neoplasm have a 4-fold increased risk of advanced neoplasm. Furthermore, smoking and non-alcoholic fatty liver disease increases the risk of CRC [7].

CRC are easily prevented through CRC screening which can detect the disease during its early stages when the survival rates are high [17]. In order to create targeted messages and educational material aimed to booster participation in screening, it is required to understand the levels of knowledge, attitudes and beliefs of the target population [18]. Poor knowledge of CRC risk factors and the benefits of screening programs are potential barriers towards active participation in screening campaigns [19]. Previous researches suggested that the level of knowledge of CRC was poor both in developed and developing countries; with even lower levels of knowledge about the well-established screening tools and their benefits $[17,20,21]$. The current work aimed to determine the level of knowledge, attitude and practice of CRC and its risk factors among mall adult visitors in Riyadh, Saudi Arabia.

\section{Subjects and Methods}

\subsection{Study Design and Study Population}

A quantitative, observational, cross-sectional study design was approved by Institutional Review Board (IRB) of King Saud University (KSU). The study participants were 18 years old or more, attending five randomly selected malls in Riyadh city, KSA.

\subsection{Sample Size}

For sample size calculation, we assumed that the knowledge should be about $18 \%$ among the study cohort. This has been assumed from a previous study on the level of knowledge among Saudis about cancer [22]. An estimated sample of 236 subjects was calculated according to the WHO sample size calculator for descriptive study designs [23]. This was multiplied by the design effect (DEFF) of two to compensate for non-simple random sampling [23]. To compensate for nonresponse 100 participants have been added. A total of 600 participants (120 participants from each mall) were recruited.

\subsection{Sampling Technique}

A stratified random sampling technique was conducted in five different regions of Riyadh city. The regions were categorized as South, West, North, Middle and East. This was followed by random selection of one mall from each region. The total sample size was 600 participants (120 from each mall).

\subsection{Data Collection}

A modified validated questionnaire was adopted for the purpose of this study [24]. It was composed of five sections; demographics, knowledge, attitude, practice and information about CRC. Data collectors were responsible for full explanation of the study aims, methods and benefits. Then they started distributing the questionnaire to the selected mall visitors, collecting them back once finished. For illiterate participants, data collectors filled the questionnaire according to their responses.

An informed consent was obtained from each subject. The consent clarified the purpose of the study, the rights of the participant to withdraw at any time without any obligation towards the study team. Participant's anonymity was assured by assigning each participant with a code number for the purpose of analysis only. Also, they were notified that any information provided will be kept confidential and will be used for the research purposes only and will not be used in any way that can identify them. No incentives or rewards was given to participants.

\subsection{Pilot Study}

A pilot study was conducted on a sample of 40 mall visitors. This pilot study was used to test the logistics of data collection, clarity of data collection tool and to estimate the timing for data collection.

\subsection{Statistical Analysis}

Data was verified and coded by the researcher. SPSS statistical software V.21 was used for data entry, management and analysis [25]. To minimize data entry errors, check codes were incorporated into the data base and the data was cleaned to ensure consistency of responses. Descriptive statistics: means, standard deviations, frequencies, percentages were calculated to the differences in the levels of KAP among the study participants.

\section{Results}

The current work was a cross-sectional study, conducted in Riyadh city in the period from May to November 2014. The 
response rate was $70 \%(n=420)$.

Table1. Socio-demographic characteristics and selected information in the study cohort.

\begin{tabular}{llcl}
\hline & & $\mathbf{n}$ & $\mathbf{\%}$ \\
\hline \multirow{2}{*}{ Gender } & Female & 229 & $55 \%$ \\
Age (years) & Male & 191 & $45 \%$ \\
Marital status & & $37 \pm 11.4(18-73)^{*}$ & \\
Education level & Married & 276 & $66 \%$ \\
& Other & 144 & $34 \%$ \\
& Illiterate & 34 & $8 \%$ \\
Family history of CRC & 1ryand2ryeducation & 168 & $40 \%$ \\
& University and above & 218 & $52 \%$ \\
Perception of personal & Yes & 32 & $8 \%$ \\
health status & No & 388 & $92 \%$ \\
& Good & 348 & $83 \%$ \\
& Uncertain & 42 & $10 \%$ \\
\hline
\end{tabular}

*Mean \pm standard deviation (Range)

Socio-demographic characteristics were summarized in table1. Women constituted $55 \%$ of the study cohort, while males constituted $45 \%$. Age of participants ranged between 18 and 73 years (mean $37 \pm 11.4$ ). About two-thirds of the study subjects were married, while half of them had university degree and above. Family history of CRC was reported in $8 \%$ of the respondents.

For the preventive measures of CRC, about a quarter (28\%) of the respondents correctly identified colonoscopy and FOBT as main CRC screening tools. Five variables were statistically significant with knowledge that colonoscopy and FOBT were the main CRC screening.

Table 2. Knowledge about CRC in the study population.

\begin{tabular}{llll}
\hline & \multicolumn{2}{l}{ Correctly answered } & Do not know \\
\cline { 2 - 4 } & Yes(\%) & No(\%) & (\%) \\
\hline Definition of CRC & 34 & 21 & 45 \\
Risk Factors & & & \\
Red meat & 16 & 84 & --------- \\
Physical inactivity & 27 & 73 & -------- \\
High caloric fatty meal & 36 & 64 & -------- \\
Cigarette smoking & 39 & 61 & ------- \\
Family history of CRC & 38 & 62 & ------- \\
Colon polyps & 17.5 & 82.5 & -------- \\
Colon ulcer & 44 & 56 & -------- \\
Colon inflammation & 34 & 66 & -------- \\
Non risk factors & & & \\
Iron deficiency & 92 & 8 & --------- \\
Diabetes mellitus & 95 & 5 & --------- \\
Oral contraceptive pills & 95.5 & 4.5 & --------- \\
Hypertension & 92 & 8 & --------- \\
Fruit and Vegetables intake & 97 & 3 & --------- \\
Aspirin medication & 97 & 3 & -------- \\
Preventive measures & & & \\
Colonoscopy & 56 & 5 & 34 \\
FOBT & 32 & 10 & 49 \\
DCBE(enema) & 5 & 9 & 72 \\
Non preventive measures & & & \\
Blood tests & 10 & 32 & 56 \\
Abdominal ultrasound & 9 & 55 \\
\hline
\end{tabular}

Table 2 depicted the participant's level of knowledge about $\mathrm{CRC}$ definition, risk factors as well as the most common prevention measures. An evaluation of the results revealed poor levels of knowledge as the majority of respondents provided the wrong answers. Only one-third of the study respondents were able to give correct definition of CRC and a wide range of responses were given regarding the factors believed as potentially CRC determinants.

Regarding the knowledge about CRC risk factors, more than one-third of the participants identified cigarette smoking, family history of CRC and inflammatory bowel disease (IBD) as risk factors for CRC. Moreover, less than one-fifth (18\%) identified colorectal polyps and red meat intake as risk factors for the disease. However, more than $90 \%$ of the respondents believed that intake of fruit and vegetables, diabetes mellitus, Aspirin medication and iron deficiency should not be risk factors for CRC. In addition, two main modifiable risk factors (high caloric intake from fat and low physical activity) were correctly identified by only $16 \%$ of the study cohort.

Table 3. Attitude about CRC in the study cohort.

\begin{tabular}{l|ll}
\hline & N & \% \\
\hline Risk perception of contracting CRC & & \\
High & 43 & 10 \\
Not known & 247 & 59 \\
Low & 130 & 31 \\
Utility of screening test for CRC prevention & & \\
Useful & 295 & 70 \\
Uncertain & 103 & 25 \\
Useless & 21 & 5 \\
\hline
\end{tabular}

As regard the level of attitude towards the risk of CRC. The mean score reported by the respondents was $2.6 \pm 0.9$ with $90 \%$ of participants either have no idea or low perception. This was an indication for the low level of perception of the disease risk. In respect to the utilization of screening tools for CRC, Only $5 \%$ of the respondents reported that the utilization of screening tools is useless in the disease detection, management and outcome. However, more than two-thirds of responses indicated that screening tools are useful in CRC prevention and control (Table3).

Table 4. Behavior of participants regarding CRC prevention.

\begin{tabular}{l|ll}
\hline Variables & No. & $\mathbf{\%}$ \\
\hline In the last year, have you modified your dietary habits for fear of & \\
contracting CRC & 116 & 28 \\
Yes & 304 & 72 \\
No & \multicolumn{2}{l}{} \\
In the last year, have you modified your physical activity for fear of \\
contracting CRC & 85 & 20 \\
Yes & 335 & 80 \\
No & \multicolumn{2}{|l}{} \\
Fecal occult blood test & 28 & 7 \\
Yes & 392 & 93 \\
No & 23 & 5 \\
Colonoscopy & 397 & 95 \\
Yes & & \\
No
\end{tabular}

Regarding behavior, the majority of respondents declared that they did not change their diet for fear of contracting CRC, while about $28 \%$ of participants modified their dietary 
habits. Likewise, only $20 \%$ of the study cohort modified their physical activity to guard against the disease.

As regards the practice of screening for CRC; as low as $7 \%$ of respondents had fecal occult blood test and only 5\% had colonoscopy as shown in (table4).

\section{Discussion}

The current study focused on a segment of Saudi adults based in Riyadh city in the Kingdom of Saudi Arabia in relation to their level of knowledge and attitude as well as their preventive practices in reference to CRC. As such, the findings of the study will be crucial to policy makers and educators, particularly in efforts geared towards holding preventive campaigns.

This study focused on the younger population and the sample selection was justified as follows; the younger generation was deemed to require special preference given that their prior behaviors as targets of promotion and information resulted in positive impacts on public health. Furthermore, this was likely to be replicated in reference to CRC, thus increasing the target population's knowledge and understanding about the disease, hence the embrace of prevention measures such as screening tests.

Overall, the study findings revealed a general lack of knowledge and understanding about CRC, hence identifying important gaps in literature. Drawing from the responses of the individual items, only one-third of the target population was able to correctly define CRC. Another crucial finding in relation to the same concept was that only $16 \%$ of the respondents answered correctly about red meat being one of the risk factors for CRC, $44 \%$ of the respondents answered correctly about colon ulcer being one of the risk determinants.

Regarding screening tools, about half of the study group correctly identified colonoscopy and $32 \%$ correctly identified FOBT as screening tests for prevention of the disease.

In comparison to other countries, similar low levels of knowledge about prevention of CRC have been reported [17,20-22]. For example in Italy, a target population of adults identified colonoscopy and FOBT as main screening tests for prevention of CRC at $62 \%$ and $52 \%$ respectively [24]. In respect to low physical activity and polyps as risk factors, there were identified by $24 \%$ and $63 \%$ respectively [24]. Findings of this study revealed that male respondents were more likely to give correct answers in relation to the main risk factors. This was consistent with other studies carried out in the KSA $[9,20,22]$. On the other hand, contradictory results were reported in a study in the USA where females had higher levels of knowledge about CRC [21].

Other variables that influenced correct answers included higher educational levels, family history of $\mathrm{CRC}$, those practicing regular physical activity and modified it due to the fear of contracting the disease as well as those who knew the correct definition of it. These findings were in agreement with the majority of previous studies carried out to explore the correlates of knowledge about cancer in general and CRC in particular, among general population, worldwide

\section{$[20,21,26,27]$}

Consequently, the study confirmed that those with a good perception of their health status were more likely to provide the right answers for questions related to screening tests. Ultimately, it was identified that information of CRC was not well disseminated. Similar findings were identified in other studies in the KSA $[9,22]$ and other countries [20,21].

Our findings concerning attitudes towards both the prevention of CRC and its screening tests in particular were encouraging, with $70 \%$ of the cohort reported that they have utilized screening tests before. Only 3\% of participants showed concern about the risk of developing the disease, which might indicate (a low level of knowledge). This was in accordance with previous studies conducted among Saudi general population [9,22].

The findings of the current work showed that $37 \%$ of participants believed that it is possible to prevent CRC, this can have implication on designing and implementing health campaigns to address the point of preventability of the disease. On the other hand, $77 \%$ of participants believed that $\mathrm{CRC}$ can be treated if diagnosed early, which is a good sign in helping to motivate people with implementing screening programs. This was consistent with other national and international studies [13,18,19,24].

The benefit of a screening test was affected by the need for additional information, this goes with previous point discussed. Knowing the definition of CRC and believed that it can be treated if diagnosed at early stage were significant in having a positive attitude towards CRC screening test. The last two points added to the previous were that if attitude towards CRC needed to be improved, the knowledge of the target population need to be improved through variable methods. The current results showed that participants needed more information about the disease, which resulted in better attitude towards its screening.

Preventive practices were associated with being a male, having family history of CRC, having a good knowledge of correct screening tests and perceived a high risk of the disease. The previous findings can be explained by the notion that this group had better knowledge than the rest of sample. As it was shown, knowledge and perceived risk of CRC resulted in adopting preventive practices.

From the results, both screening tests were adopted by half the participants who undergone screening, there was no difference in doing FOBT or colonoscopy which may indicate that the current practice of colorectal screening in the kingdom needed enforcement through the raise of awareness about the importance of these tools for disease prevention and hence management. Consistent findings were identified in other previous researches [9,14,17-19,22].

\section{StudyStrength and Limitation}

The results however had encountered several limitations; the study was conducted on a cross-section of the society and could not be taken as the entire truth. The responses were often incomplete as they are self-administered questionnaire 
(subjective responses). However, such a possibility is low as incomplete responses were quite few in number and they were reported personally in confidence and in a nun known setting. This was common for such studies where responses may have been limited and hence the answers of those who are knowledgeable participants are sure to be different from those who did not answer correctly. Notwithstanding, even if the response rate was low, the validity of the data gathered from such surveys cannot be ignored and it does not affect the actual picture of the situation but one cannot come to any general conclusions.

\section{Conclusion}

In conclusion, it can be said that the data available from the conducted study on different levels of knowledge, attitude and practice about CRC among Saudi people, the more educated individuals, those aware of family history of $\mathrm{CRC}$, its detection and prevention and those who are not so aware of it.

The main point of the current work was the need for implementation of awareness programs to establish a strong link between the public, health centers and educational institutions to spread the awareness of CRC. Hence, presentation of a clear picture of the disease, its detection and more emphasis should be on the screening tests required for the prevention of disease at all levels of individual.

\section{References}

[1] Lozano, R., et al., Global and regional mortality from 235 causes of death for 20 age groups in 1990 and 2010: a systematic analysis for the Global Burden of Disease Study 2010. Lancet, 2012. 380(9859): p.2095-128.

[2] WHO, World Cancer Report 2014, in International Agency for Research on Cancer. 2014, WHO: Geneva.

[3] Merika, E., et al., Review. Colon cancer vaccines: an update. In vivo, 2010. 24(5): p.607-28.

[4] Ferlay, J., et al., Colorectal Cancer Incidence, Mortality and Prevalence Worldwide in 2008. Int J Cancer, 2010. 127(12): p.2893-917.

[5] Giovannucci, E., Modifiable risk factors for colon cancer. Gastroenterologyclinics of North America, 2002. 31(4): p.925-43.

[6] Siegel, R., C. Desantis, and A. Jemal, Colorectal cancer statistics, 2014. CA: a cancer journal for clinicians, 2014. 64(2): p.104-17.

[7] Ng, S. and S. Wong, Colorectal cancer screening in Asia. British medical bulletin, 2013. 105(1): p.29-42.

[8] Sung, J., et al., Increasing incidence of colorectal cancer in Asia: implications for screening. The lancet oncology, 2005. 6(11): p.871-76.

[9] Mosli, M. and M. Al-Ahwal, Colorectal cancer in the Kingdom of Saudi Arabia: need for screening. APJCP, 2012. 13(8): p.3809-813.
[10] Al-Eid, H., Cancer Incidence Report 2010, in Saudi Cancer Registry, S. Bazarbashi, Editor. 2014, Ministry of Health: Kingdom of Saudi Arabia.

[11] Mandel, J., T. Church, and J. Bond, The effect of fecal occultblood screening on the incidence of colorectal cancer. NEJM, 2000. 343(22): p.1603-07.

[12] Mack, L., H. Stuart, and W. Temple, Survey of colorectal cancer screening practices in a large Canadian urban centre. Canadian Journal of Surgery, 2004. 47(3): p.189-94.

[13] Federici, A., et al., Survey on colorectal cancer screening knowledge, attitudes, and practices of general practice physicians in Lazio, Italy. Preventive Medicine, 2005. 41(1): p.30-35.

[14] Misra, S., et al., Cost effectiveness of interventions to promote screening for colorectal cancer: a randomized trial. JPMPH, 2011. 44(3): p.101-10.

[15] Arbyn, M., et al., European Commission's proposal for a council recommendation on cancer screening. BMJ, 2003. 327(7409): p.289-90.

[16] Ibrahim, E., et al., Past, present and future of colorectal cancer in the Kingdom of Saudi Arabia. Saudi journal of gastroenterology, 2008. 14(4): p.178-82.

[17] Christou, A. and S. Thompson, Colorectal cancer screening knowledge, attitudes and behavioural intention among Indigenous Western Australians. BMC Public Health 2012. 12(528).

[18] Deutekom, M., et al., Uptake of faecal occult blood test colorectal cancer screening by different ethnic groups in the Netherlands. Eur J Publ Health, 2009. 19(4): p.400-02.

[19] Worthley, D., et al., Screening for colorectal cancer by faecal occult blood test: why people choose to refuse. Intern Med J, 2006. 36(9): p.607-10.

[20] Puteh, S., et al., Relationship of Knowledge, Attitude, Practice (KAP) and Demographic Factors with Quality of Life among Urban Colorectal Cancer Patients in Malaysia. Middle East Journal of Cancer 2014. 5(1): p.31-41.

[21] Aparna, K., The Knowledge, Attitudes and Practices Approach: an evaluation of the barriers to colorectal cancer screening at Orange Blossom Family Health Johns Hopkins University School of Medicine, 2014. PCLPSite: Health Care Center for the Homeless, Orange Blossom Family Health, Orlando,.

[22] Ravichandran, K., N. Al-Hamdan, and G. Mohamed, Knowledge, attitude, and behavior among Saudis toward cancer preventive practice. J Family Community Med, 2011. 18(3): p.135-42.

[23] WHO, sample size and power, in An epidemiological approach to reproductive health, P. Wingo, et al., Editors. 1994, WHO: Geneva, Switzerland. p.16-57.

[24] Sessa, A., et al., Knowledge, attitudes, and preventive practices about colorectal cancer among adults in an area of Southern Italy. BMC cancer, 2008. 8(171).

[25] IBM Corp. Released 2012. IBM SPSS Statistics for Windows VA, N.I.C. 
[26] Al-Amri, F. A., et al., Breast cancer correlates in a cohort of breast screening program participants in Riyadh, KSA. Journal of the Egyptian National Cancer Institute, 2015. 27(2): p. $77-82$.
[27] Saeedi, M., et al., Knowledge, attitude and practice of breast cancer screening among female general practitioners in Riyadh, Saudi Arabia. Cancer Research Journal 2014. 2(6): p.108-13. 